\title{
A!
}

This is an electronic reprint of the original article.

This reprint may differ from the original in pagination and typographic detail.

Harnefors, Lennart; Schweizer, Mario; Kukkola, Jarno; Routimo, Mikko; Hinkkanen, Marko; Wang, Xiongfei

\section{Generic PLL-Based Grid-Forming Control}

Published in:

IEEE Transactions on Power Electronics

DOI:

10.1109/TPEL.2021.3106045

Published: 01/02/2022

Document Version

Peer reviewed version

Please cite the original version:

Harnefors, L., Schweizer, M., Kukkola, J., Routimo, M., Hinkkanen, M., \& Wang, X. (2022). Generic PLL-Based Grid-Forming Control. IEEE Transactions on Power Electronics, 37(2), 1201-1204.

https://doi.org/10.1109/TPEL.2021.3106045

This material is protected by copyright and other intellectual property rights, and duplication or sale of all or part of any of the repository collections is not permitted, except that material may be duplicated by you for your research use or educational purposes in electronic or print form. You must obtain permission for any other use. Electronic or print copies may not be offered, whether for sale or otherwise to anyone who is not an authorised user. 


\title{
Generic PLL-Based Grid-Forming Control
}

\author{
Lennart Harnefors, Fellow, IEEE, Mario Schweizer, Member, IEEE, Jarno Kukkola, Mikko Routimo, Member, \\ IEEE, Marko Hinkkanen, Senior Member, IEEE, and Xiongfei Wang, Senior Member, IEEE
}

\begin{abstract}
In grid-forming control, the swing equation of a synchronous machine is emulated by a power controller. Thereby, frequency droop, power-oscillation damping, and/or virtual inertia can be obtained. In this letter it is shown that a phaselocked loop (PLL) — which is normally grid following-can be designed so as to, in turn, emulate a generic power controller, thus, becoming grid forming.
\end{abstract}

Index Terms-Grid-connected converters, grid-forming control, phase-locked loops, voltage-source converters.

\section{INTRODUCTION}

G RID-FORMING CONTROL of a voltage-source converter has two fundamental properties.

- Property 1: The back electromotive force of a synchronous machine is emulated, giving a stiff converter voltage.

- Property 2: The swing equation of a synchronous machine is emulated, giving an active-power response to grid-frequency variations.

Schemes based on vector current control are normally grid following. Yet, in [1], it is shown that Property 1 can be fulfilled by a vector-current-control scheme, designed for correspondence to power-synchronization control (PSC).

Property 2 is, in conventional PSC, fulfilled by a power controller (PC) [2]. In [1], a hybrid synchronization controller (SC) is proposed, where the PC is combined with a phaselocked loop (PLL). A similar scheme is suggested in [3]. In PSC, the PLL is needed for synchronization at startup [2] as well as to prevent loss of synchronism when converter-current limitation is applied [4]. During normal operation, the PLL can be disengaged and the PC solely be relied upon.

Unfortunately, as a consequence, during current limitation, an on/off chattering behavior of the PLL may occur. One way of preventing this would be somehow to make the PLL fulfill Property 2, allowing the PC to be eliminated, thus, simplifying the structure of the SC. Steps in that direction have been taken. A PLL is in [5] shown to have structural similarities to the swing equation, and can reproduce certain characteristics thereof if tuned properly [6], [7].

This work was supported in part by $\mathrm{ABB}$

L. Harnefors is with $\mathrm{ABB} \mathrm{AB}$, Corporate Research, 72226 Västerås, Sweden (e-mail: lennart.harnefors@se.abb.com).

M. Schweizer is with ABB Switzerland Ltd, Corporate Research, 5405 Baden-Dättwil, Switzerland (e-mail: mario.schweizer@ch.abb.com).

J. Kukkola and M. Hinkkanen are with the Department of Electrical Engineering and Automation, Aalto University, 02150 Espoo, Finland (e-mail: jarno.kukkola@aalto.fi, marko.hinkkanen@aalto.fi).

M. Routimo is with ABB Oy Drives, 00380 Helsinki, Finland (e-mail: mikko.routimo@fi.abb.com).

X. Wang is with the Department of Energy Technology, Aalborg University, 9100 Aalborg, Denmark (e-mail: xwa@et.aau.dk).

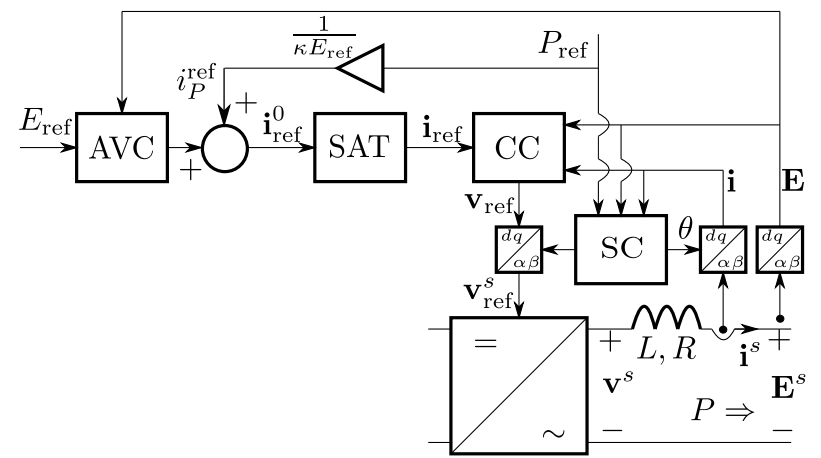

Fig. 1. Circuit and controller block diagram.

The contribution of this letter-in relation to prior art, where certain special cases are considered-is the design of a PLL-based grid-forming control (PLL-GFC) scheme that emulates a generic PC. ${ }^{1}$ See Section III. The emulation is not exact, but the accuracy is sufficient as seen from the fairly slow time scale of grid-frequency variations. Thus, Property 2 is fulfilled. Design for PSC correspondence, fulfilling also Property 1, is considered in Section IV. (The design in Section IV summarizes the key results of [1], but PC emulation by the PLL is not considered in [1].) Experimental evaluation with performance comparison of PLL-GFC and PSC is made in Section V.

\section{Preliminaries}

Boldface letters denote complex space vectors. The superscript $s$ denotes a vector referred to the stationary $\alpha \beta$ reference frame. The corresponding vector referred to the synchronous $d q$ reference frame, which has angle $\theta$ as reference, is denoted without a superscript. Italic letters denote scalar variables and real transfer functions. The reference for a controlled variable is denoted by appending the sub- or superscript ref. The Laplace variable $s$ is to be considered as the operator $s=d / d t$, where appropriate.

Fig. 1 illustrates the main circuit and the controller block diagram. The former consists of an inductor, with inductance $L$ and inner resistance $R$, between the converter and point-ofcommon-coupling (PCC) buses. The respective voltage vectors are $\mathbf{v}^{s}$ and $\mathbf{E}^{s}$, whereas the output current is $\mathbf{i}^{s}$. The active output power at the PCC is calculated as

$$
P=\kappa \operatorname{Re}\left\{\mathbf{E}^{s}\left(\mathbf{i}^{s}\right)^{*}\right\}=\kappa \operatorname{Re}\left\{\mathbf{E i}^{*}\right\}, \quad \kappa=\frac{3}{2 K^{2}}
$$

\footnotetext{
${ }^{1}$ Important special cases of the generic PC are: pure proportional, giving frequency droop; proportional-integral, giving power-oscillation damping and virtual inertia; and lead-lag, giving frequency droop, power-oscillation damping, and virtual inertia [8].
} 


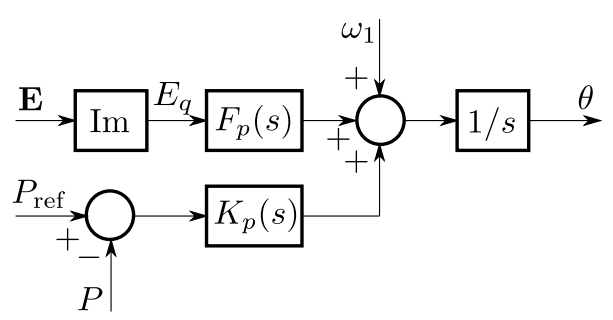

Fig. 2. Hybrid SC, combining a PLL with controller $F_{p}(s)$ with a PC $K_{p}(s)$.

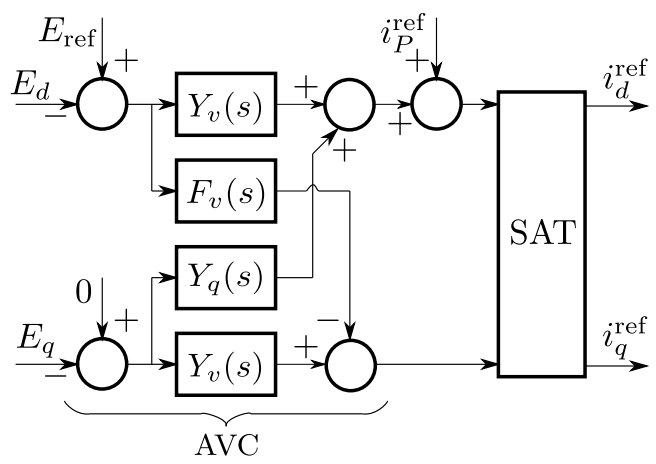

Fig. 3. Generalized AVC with current-reference selection and SAT.

where the superscript $*$ denotes complex conjugate and $K$ is the space-vector scaling constant. The $d q$ frame is statically aligned with the $\mathrm{PCC}$ voltage, so that $\mathbf{E}=E$ (i.e., real), giving $P=\kappa E i_{d}$ in the steady state.

Voltage reference $\mathbf{v}_{\text {ref }}$ is computed by a current controller (CC). The ideal current reference is set as

$$
\mathbf{i}_{\mathrm{ref}}^{0}=i_{P}^{\mathrm{ref}}+\operatorname{AVC}\left(E_{\mathrm{ref}}, \mathbf{E}\right), \quad i_{P}^{\mathrm{ref}}=\frac{P_{\mathrm{ref}}}{\kappa E_{\mathrm{ref}}}
$$

where the second term is the complex output of a generalized ac-bus-voltage controller (AVC), see Section III for details. To avoid absolute values exceeding the maximum permissible current, vector saturation (SAT) is then applied, forming $\mathbf{i}_{\text {ref }}=$ $\operatorname{SAT}\left(\mathbf{i}_{\text {ref }}^{0}\right)$. The CC and the AVC are assumed to be tuned such that the resulting closed-loop step-response rise times are in the ranges of tens and hundreds of milliseconds or less, respectively. In addition, zero static control errors are assumed, i.e., $\mathbf{i}=\mathbf{i}_{\text {ref }}$ and $E_{d}=E_{\text {ref }}$ statically. The latter achieved by the AVC adjusting $i_{q}^{\text {ref }}$.

\section{MAin Result}

Fig. 2 shows the hybrid SC of [1], where $\omega_{1}$ is the nominal angular grid frequency. The objective is now to make the PLL emulate the PC, i.e., the signal path through $F_{p}(s)$ should have a similar effect [for $K_{p}(s)=0$ ] as the signal path through $K_{p}(s)$ [for $F_{p}(s)=0$ ]. Clearly then, a mechanism that forces the PLL input signal $E_{q}$ to be proportional to the PC input signal $P_{\text {ref }}-P$ is needed. A static relation between the two input signals cannot be obtained, so an exact emulation is impossible. However, a quasi-stationary relation, which captures the dynamics of grid-frequency variations well enough, can be obtained, as will now be demonstrated.

Since active power, according to (1), is produced by $i_{d}$, the first step is to create a coupling from $E_{q}$ to $i_{d}^{\text {ref }}$, in turn, giving a coupling to $P$. This can be accomplished by implementing (2) as shown in Fig. 3, in equation form

$$
\begin{aligned}
\operatorname{AVC}\left(E_{\mathrm{ref}}, \mathbf{E}\right)= & Y_{v}(s)\left(E_{\mathrm{ref}}-\mathbf{E}\right)-Y_{q}(s) E_{q} \\
& -j F_{v}(s)\left(E_{\mathrm{ref}}-E_{d}\right) .
\end{aligned}
$$

Here, $F_{v}(s)$ is a conventional AVC, coupling from $E_{d}$ to

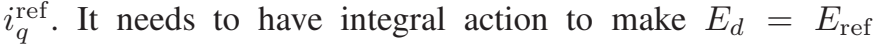
statically. $Y_{v}(s)$ is an active damper consisting of a gain (active conductance) $G_{a}$ in cascade with a low-pass filter. It is vital for fulfilling Property 1 , see Section IV. $Y_{q}(s)$ similarly consists of a gain (active susceptance) $B_{a}$ in cascade with a low-pass filter. It creates the required coupling from $E_{q}$ to $i_{d}^{\text {ref }}$.

Let us now analyze the impact of (3) seen from the time scale of grid-frequency variations. This time scale is given by the characteristic inertia constant of the grid, which is typically in the range of seconds. This is substantially slower than the closed-loop-system rise times assumed in Section II. Consequently, in the time scale of grid-frequency variations, the static relations $\mathbf{i}=\mathbf{i}_{\text {ref }}$ and $E_{d}=E_{\text {ref }}$ can be considered. In addition, the filters in $Y_{v}(s)$ and $Y_{q}(s)$ are assumed to have high enough bandwidths to be considered statically as well, i.e., $Y_{v}(0)=G_{a}$ and $Y_{q}(0)=B_{a}$. Assuming that SAT is not effectuated, (2) and (3) then yield

$$
\mathbf{i}=i_{P}^{\mathrm{ref}}-B_{a} E_{q}-j \underbrace{\left(G_{a} E_{q}+i_{v}\right)}_{-i_{q}}
$$

where $i_{v}$ is the quasi-static value of the integrator of the conventional AVC. Substitution in (1) yields

$$
P=\kappa \operatorname{Re}\left\{\mathbf{E i}^{*}\right\}=P_{\text {ref }}-\kappa\left(E_{\text {ref }} B_{a}-i_{q}\right) E_{q} .
$$

Solving for $E_{q}$ in (5) gives the desired proportionality between the PC and PLL input signals as

$$
E_{q}=\frac{1}{\kappa\left(E_{\mathrm{ref}} B_{a}-i_{q}\right)}\left(P_{\mathrm{ref}}-P\right) .
$$

Consequently, for the PLL to emulate the PC, the PLL controller should be selected as

$$
F_{p}(s)=\kappa\left(E_{\mathrm{ref}} B_{a}-i_{q}^{f}\right) K_{p}(s)
$$

where $i_{q}^{f}$ is a low-pass filtering of $i_{q}$ (or, if preferred, $i_{q}^{\text {ref }}$ ). Low-pass filtering is desired to suppress noise in the variable gain of (7).

The working principle of the proposed scheme is perhaps easiest understood by expressing the PCC voltage in polar form as $\mathbf{E}^{s}=E e^{j \theta_{p}} \Rightarrow \mathbf{E}=E e^{j \Delta \theta}, \Delta \theta=\theta_{p}-\theta$, giving $E_{q}=E \sin \Delta \theta$. Suppose that $\Delta \theta=0$ initially. A variation in the grid frequency, let us say a drop, results in $\theta_{p}$ lagging $\theta$, i.e., $\Delta \theta$ becomes negative. Via the second term in (3), this causes an active-power injection. The characteristics thereof are determined solely by $K_{p}(s)$; due to the factor $\kappa\left(E_{\mathrm{ref}} B_{a}-i_{q}^{f}\right)$ in (7), the characteristics are invariant of $B_{a}$. A larger $B_{a}$ merely results in smaller transients in $\Delta \theta$ and, thus, in $E_{q}$. At the very least, to avoid the risk that the gain of (7) changes sign, $B_{a}>\max \left(i_{q}\right) / E_{\text {ref }}$ should be selected. Normally, $\max \left(i_{q}\right) / E_{\text {ref }} \leq 1$ per unit (p.u.). 


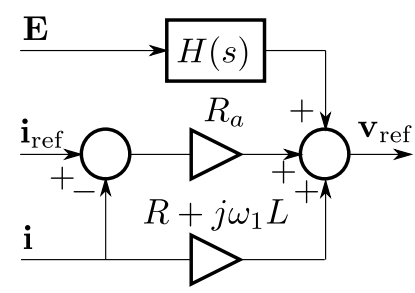

Fig. 4. Proportional-decoupling-feedforward CC.

TABLE I

CONTROLLER SELECTIONS

\begin{tabular}{c|c|c}
\hline & PSC & PLL-GFC \\
\hline$K_{p}(s)$ & $K_{p 0}(s)$ & 0 \\
$F_{p}(s)$ & 0 & $\kappa\left(E_{\mathrm{ref}} B_{a}-i_{q}^{f}\right) K_{p 0}(s)$ \\
$Y_{v}(s)$ & $\frac{1}{R_{a}}\left(1+\frac{\alpha_{a}}{s}\right) H(s)$ & $\frac{1}{R_{a}} H(s)$ \\
$F_{v}(s)$ & 0 & $\frac{\kappa K_{p 0}(s) E_{\mathrm{ref}}^{2} H(s)}{R_{a}^{2} s}$ \\
$Y_{q}(s)$ & 0 & $B_{a} H(s)$ \\
\hline
\end{tabular}

\section{DESIGN FOR PSC CORRESPONDENCE}

As long as the assumptions relied upon in Section III are fulfilled, the CC and blocks $Y_{v}(s)$ and $F_{v}(s)$ of the AVC can be chosen freely. However, of particular interest is a design giving near equivalence between PLL-GFC and the slightly modified PSC variant considered in [1], thus, ensuring that Property 1 is fulfilled. As shown in [1], such a design is obtained by using a proportional-decoupling-feedforward CC (see Fig. 4)

$$
\mathbf{v}_{\text {ref }}=R_{a}\left(\mathbf{i}_{\text {ref }}-\mathbf{i}\right)+\left(R+j \omega_{1} L\right) \mathbf{i}+H(s) \mathbf{E} .
$$

In the PCC-voltage feedforward term, the recommended lowpass filter selection is $H(s)=\alpha_{c} /\left(s+\alpha_{c}\right)$, where $\alpha_{c}=R_{a} / L$ is the bandwidth of the closed current control loop that results from (8).

With $K_{p 0}(s)$ as the desired PC (to be implemented in PSC and emulated in PLL-GFC), Table I shows the controller selections, with reference to Figs. 2 and 3, for PSC and PLLGFC, respectively. The following should be noted (see [1] for details).

- To fulfill Property 1 , the active conductance is selected as $G_{a}=1 / R_{a}$.

- PSC uses neither a conventional AVC, nor a PLL. To ensure that $\mathbf{E}=E_{\text {ref }}$ statically despite that, an integral part (with gain $\alpha_{a}$ ) is included in $Y_{v}(s)$.

- For PLL-GFC, in addition to $F_{p}(s)$, also $F_{v}(s)$ is made proportional to $K_{p 0}(s)$. Under idealized conditions, this gives the same small-signal dynamics as the PC.

- For simplicity, the low-pass filter in $Y_{q}(s)$ is chosen identical to that in $Y_{v}(s)$.

\section{EXPERIMENTAL AND Simulation Results}

The experimental setup-see Table II for data-uses backto-back converters, allowing dc-bus-voltage control from the converter not being studied. There is a shunt capacitor (with capacitance $C$ ) at the PCC, forming an inductive-capacitiveinductive filter with the grid inductance $L_{g}$ behind the gridvoltage source.
TABLE II

EXPERIMENTAL SETUP DATA

\begin{tabular}{|c|c|c|}
\hline Variable/parameter & Actual value & Normalized value \\
\hline Rated power & $12.5 \mathrm{kVA}$ & 1 p.u. \\
\hline Rated voltage & $\sqrt{2 / 3} \cdot 400 \mathrm{~V}$ & 1 p.u. \\
\hline Rated current & $\sqrt{2} \cdot 18 \mathrm{~A}$ & 1 p.u. \\
\hline Maximum current & $1.5 \cdot \sqrt{2} \cdot 18 \mathrm{~A}$ & 1.5 p.u. \\
\hline Base impedance & $12.8 \Omega$ & 1 p.u. \\
\hline Filter inductance $L$ & $3.3 \mathrm{mH}$ & 0.081 p.u. \\
\hline Filter resistance $R$ & $0.51 \Omega$ & 0.040 p.u. \\
\hline Filter capacitance $C$ & $8.8 \mu \mathrm{F}$ & 0.036 p.u. \\
\hline Fundamental frequency & $50 \mathrm{~Hz}$ & 1 p.u. \\
\hline Rated dc-bus voltage & $650 \mathrm{~V}$ & 2 p.u. \\
\hline Sampling frequency & $10 \mathrm{kHz}$ & 200 p.u. \\
\hline Switching frequency & $5 \mathrm{kHz}$ & 100 p.u. \\
\hline
\end{tabular}

Performance comparisons of PLL-GFC and PSC, designed according to Section IV, are made. A proportional-integral PC is considered as

$$
K_{p 0}(s)=K_{p}+\frac{1}{M s} .
$$

As shown in [8], the proportional part corresponds to a poweroscillation damper and the integral part to a virtual inertia. The following p.u. parameter values are selected:

$$
\begin{aligned}
& R_{a}=0.2 \quad K_{p}=0.05 \quad M=1000 \\
& B_{a}=5 \quad \alpha_{a}=0.1 .
\end{aligned}
$$

First, $L_{g}$ is selected to get a short-circuit ratio of 1 . The grid voltage is 1 p.u. In Fig. 5, the grid frequency $\omega_{g} /(2 \pi)$ is ramped from $50 \mathrm{~Hz}$ (1 p.u.) down to $49 \mathrm{~Hz}(0.98$ p.u.) with the rate of change $5 \mathrm{~Hz} / \mathrm{s}$. As can be observed, near-identical inertial responses in $P$ are obtained from the two schemes. Due to the proportional (damping) part of (9), there are no power oscillations. The transient in $E_{q}$ for PLL-GFC results in a slightly different transient in $i_{q}$ compared to PSC.

Next, the configuration is changed to islanded operation, with zero grid voltage. $L_{g}$ is reduced to 0.5 p.u. and a 6-p.u. load resistance is put in series. $M$ is made infinite, giving a proportional-only PC, i.e., a frequency droop [8]. In Fig. 6, at $t=0.2 \mathrm{~s}$, the load resistance is decreased to 2 p.u., giving an increased power draw and, as a result, a frequency reduction. It is interesting to note that the instantaneous frequencies of PSC and PLL-GFC deviate transiently. This is because the PLL emulation of the PC is not exact. Yet, the activepower responses are virtually identical, showing that the quasistationary design principle in Section III is relevant.

The experimental setup unfortunately does not facilitate multiple converters. To show that PLL-GFC has the capability of islanded operation with multiple converters, the experimental setup is replicated in Simulink, but with two converters (I and II) connected in parallel at the PCC. The converters and their controls are identical, but to introduce an asymmetry, they have slightly different filter inductances: $L_{\mathrm{I}}=1.1 \mathrm{~L}$ and $L_{\mathrm{II}}=0.9 L$. As can be observed in Fig. 7 , the converters share the load power equally. In fact, the curves (for voltage, $E_{q}$ only) replicate the corresponding experimental curves in Fig. 6 , but scaled with $1 / 2$ due to the load sharing. 

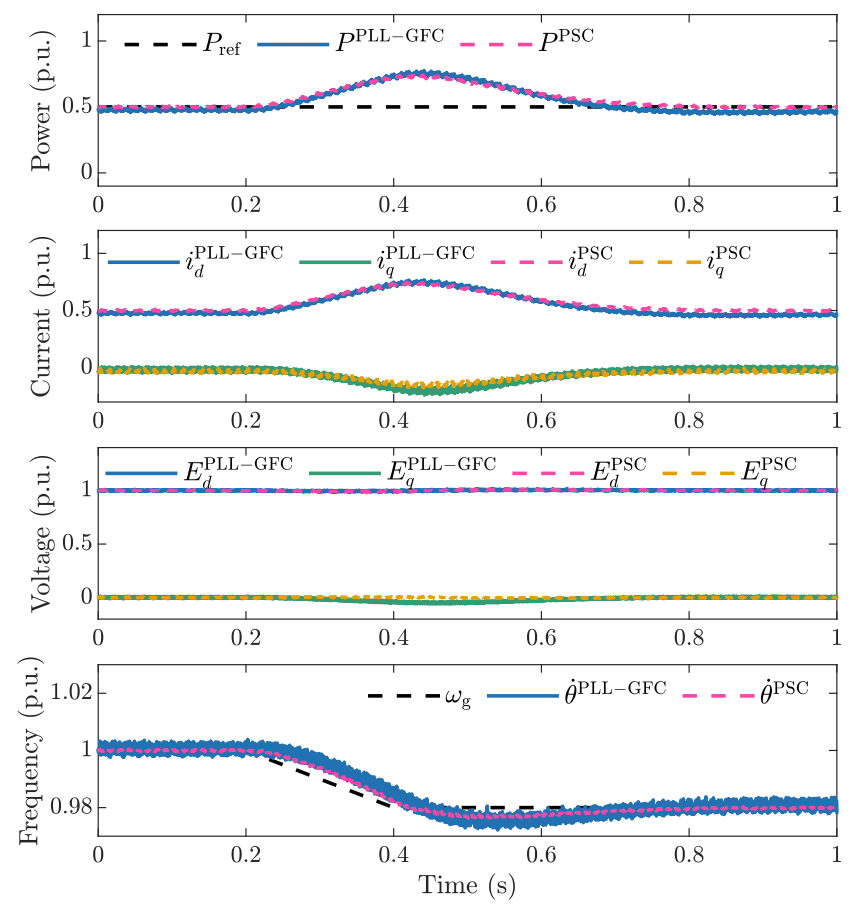

Fig. 5. Grid-frequency ramp.
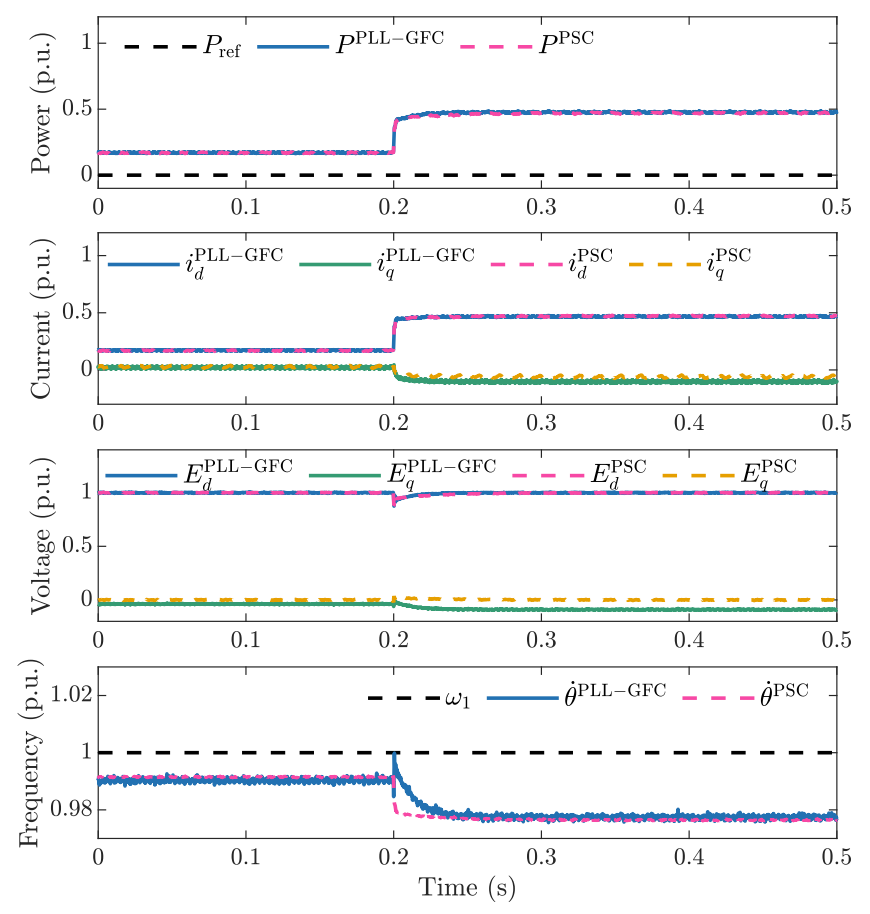

Fig. 6. Islanded operation with load-resistance step.

\section{CONCLUSION}

Previous findings concerning the similarity between a PLL and the swing equation of a synchronous machine were generalized. The scheme PLL-GFC results, where the PLL is designed to emulate a generic PC, fulfilling Property 2. Design for PSC correspondence was considered, fulfilling also Property 1.

Experimental comparison of PLL-GFC and PSC showed
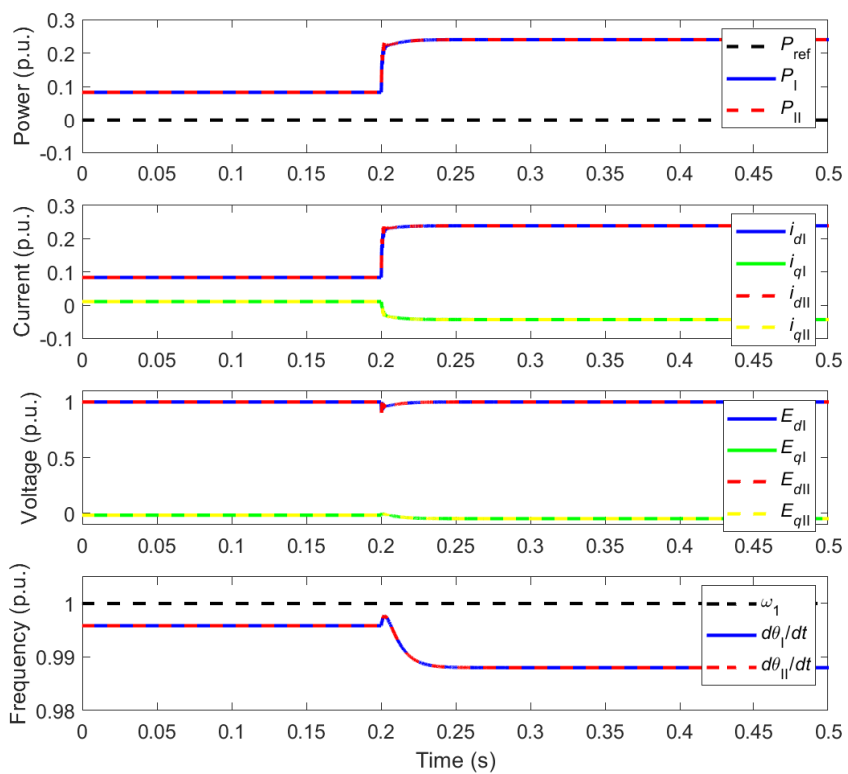

Fig. 7. Islanded operation with load-resistance step; simulation of two parallel converters (I and II) using PLL-GFC.

near identical performance. The most notable difference is that, in PLL-GFC, transients in $P$ are reflected by transients in $E_{q}$ (and vice versa). This is caused by the coupling from $E_{q}$ to $i_{d}^{\text {ref }}$, with gain $B_{a}$, introduced to fulfill Property 2 . The transients in $E_{q}$ diminish as $B_{a}$ is increased.

\section{REFERENCES}

[1] L. Harnefors, J. Kukkola, M. Routimo, M. Hinkkanen, and X. Wang, "A universal controller for grid-connected voltage-source converters," IEEE J. Emer. Sel. Top. Power Electron., Early Access, DOI: 10.1109/JESTPE.2020.3039407.

[2] L. Zhang, L. Harnefors, and H.-P. Nee, "Power-synchronization control of grid-connected voltage-source converters," IEEE Trans. Power Syst., vol. 25, no. 2, pp. 809-920, May 2010.

[3] L. Huang, H. Xin, Z. Wang, L. Zhang, K. Wu, and J. Hu, "Transient stability analysis and control design of droop-controlled voltage source converters considering current limitation," IEEE Trans. Smart Grid, vol. 10, no. 1, pp. 578-591, Jan. 2019.

[4] L. Harnefors, "Method and control system for controlling a voltage source converter using power-synchronisation control," Patent EP 3534 $522 \mathrm{~B} 1$.

[5] X. Wang, M. G. Taul, H. Wu, Y. Liao, F. Blaabjerg, and L. Harnefors, "Grid-synchronization stability of converter-based resources-An overview," IEEE Open J. Ind. Appl., vol. 1, pp. 115-134, 2020.

[6] H. Alatrash, A. Mensah, E. Mark, G. Haddad, and J. Enslin, "Generator emulation controls for photovoltaic inverters," IEEE Trans. Smart Grid, vol. 3, no. 2, pp. 996-1011, Jun. 2012.

[7] M. Zhang, X. Yuan, and J. Hu, "Inertia and primary frequency provisions of PLL-synchronized VSC HVDC when attached to islanded ac system," IEEE Trans. Power Syst., vol. 33, no. 4, pp. 4179-4188, Jul. 2018.

[8] W. Zhang, D. Remon, A. Mir, A. Luna, J. Rocabert, I. Candela, and P. Rodriguez, "Comparison of different power loop controllers for synchronous power controlled grid-interactive converters," in Proc. IEEE Energy Conv. Congr. Expos., Montreal, Sep. 2015. 